\title{
Recent results and future plans from the NA61/SHINE experiment
}

Silvestro Di Luise*

ETH Zurich Institute for Particle Physics

E-mail: silvestro.di.luise@cern.ch

NA61/SHINE (SPS Heavy Ion and Neutrino Experiment) is a fixed-target experiment to study hadron production in hadron-nucleus and nucleus-nucleus collisions at the CERN SPS. The experimental apparatus is a large acceptance Magnetic Spectrometer complemented with TimeOf-Flight detectors and a Projectile Spectator Detector. Its excellent capabilities for particle identification and momentum determination, even in complex events, makes the NA61/SHINE experiment well suited for pursuing a reach physics program which consists of three main topics:

i) hadron-nucleus interactions: hadron production measurements for neutrino (T2K) and cosmic-ray (Pierre Auger Observatory, KASCADE) experiments

ii) nucleus-nucleus interactions: hadron production measurements to search for the critical point of strongly interacting matter and to study the properties of the onset of the deconfinement

iii) proton-proton and proton-nucleus interactions: measurement of inclusive and correlated yields of high $p_{T}$ hadrons to study their in-medium modifications

In 2011/2012 numerous new physics results were released, in particular particle spectra in $p+C$ interactions at $31 \mathrm{GeV} / c$ for $\mathrm{T} 2 \mathrm{~K}$ and in $\pi^{-}+\mathrm{C}$ interactions at 158 and $350 \mathrm{GeV} / c$ for cosmic ray experiments as well as particle spectra, fluctuations and correlations in $p+p$ interactions at 20 , $31,40,80$ and $158 \mathrm{GeV} / c$ for the ion program. Results on selected topics and the NA61/SHINE future plan of measurements are briefly reported in this proceedings.

36th International Conference on High Energy Physics

4-11 July 2012

Melbourne, Australia

${ }^{*}$ Speaker. 


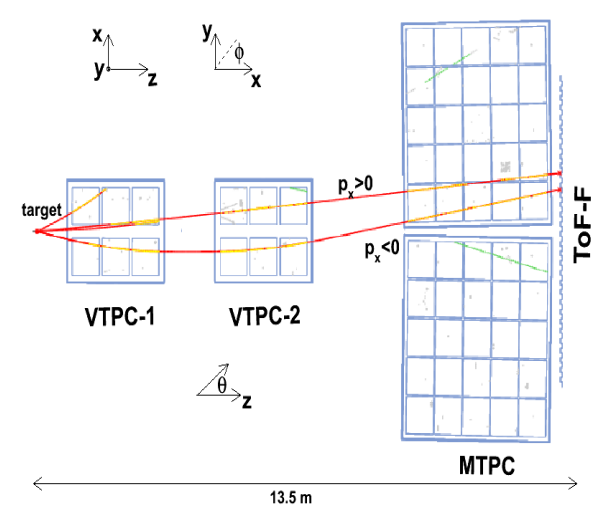

Figure 1: Schematic of the experimental apparatus and a reconstructed $p+\mathrm{C}$ event. The target, the four TPC's (VTPC and MTPC) and the Time of Flight (ToF) detector are indicated.
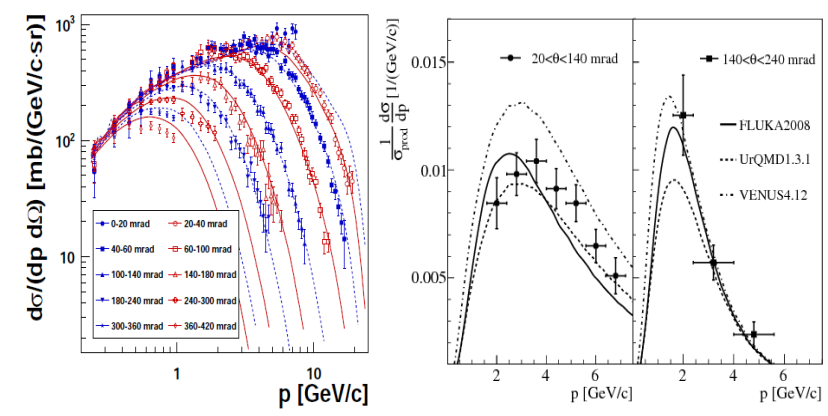

Figure 2: Production cross section of $\pi^{+}$and $K^{+}$as a function of the laboratory momentum for different intervals of the polar angle $\theta$ compared with model predictions (FLUKA2008 for $\pi$ 's). Error bars are statistical and systematic uncertainties added in quadrature. The overall uncertainty (2.3\%) due to the normalization procedure is not shown.

Results for neutrino physics. Second generation long baseline neutrino oscillation experiments, like T2K, do require a very good knowledge of neutrino fluxes [1] which means a more precise measurements of the production cross sections of hadrons like pions and kaons. NA61/SHINE (Fig. 1) thanks to the large acceptance and particle identification in the forward region covers most of the phase space region of interest for T2K. So far several results on hadron production in $p+\mathrm{C}$ interactions at $31 \mathrm{GeV} / c$ using a thin $\left(0.04\right.$ of a nuclear interaction length $\left.\lambda_{I}\right)$ graphite target have been obtained. The first set of measurements, dedicated to the determination of the $\pi^{ \pm}$and $K^{+}$ differential production cross section (Fig. 2) [2], contributed significantly to the T2K measurement of electron neutrino appearance $\left(v_{\mu} \rightarrow v_{e}\right)$ which led to the first indication of a non zero mixing angle $\theta_{13}$ and to the measurement of muon neutrino disappearance $\left(v_{\mu} \rightarrow v_{\mu}\right)$ [3]. First proton production cross sections have been released recently (Fig. 3), these data are needed to reduce the flux uncertainty related to reinteractions of secondary protons inside the T2K target. Preliminary results on neutral strange particle production are now available as well (Fig. 3). The ultimate precision on the flux prediction will finally be achieved through the measurements of hadron emission from the same (replica) target as the one used by T2K $\left(\approx 2 \lambda_{I}\right)$. NA61/SHINE has already published a pilot analysis using low statistics replica target data [4] to establish the method for re-weighting the production of pions emitted from the T2K target. Figure 3 shows the neutrino flux calculated with the re-weighting of the $\pi^{+}$production based on the replica target data compared to the flux obtained with the re-weighting based on the thin target measurements. Analysis of high-statistics thin-target and long-target data are about to be finalized.

Results for cosmic ray physics. The interpretation of extensive air shower data, as for instance recorded by the Pierre Auger Observatory and KASCADE [5], relies to large extent on the correct modeling of hadron-air interactions that occur during the shower development. In 2009, NA61/SHINE recorded data with $\pi^{-}$beams at 158 and $350 \mathrm{GeV} / c$ on a thin carbon target to provide particle-production data for the tuning of hadronic interaction models used in air-shower simulations. Preliminary results comprise first mesurements of the charged hadrons differential 

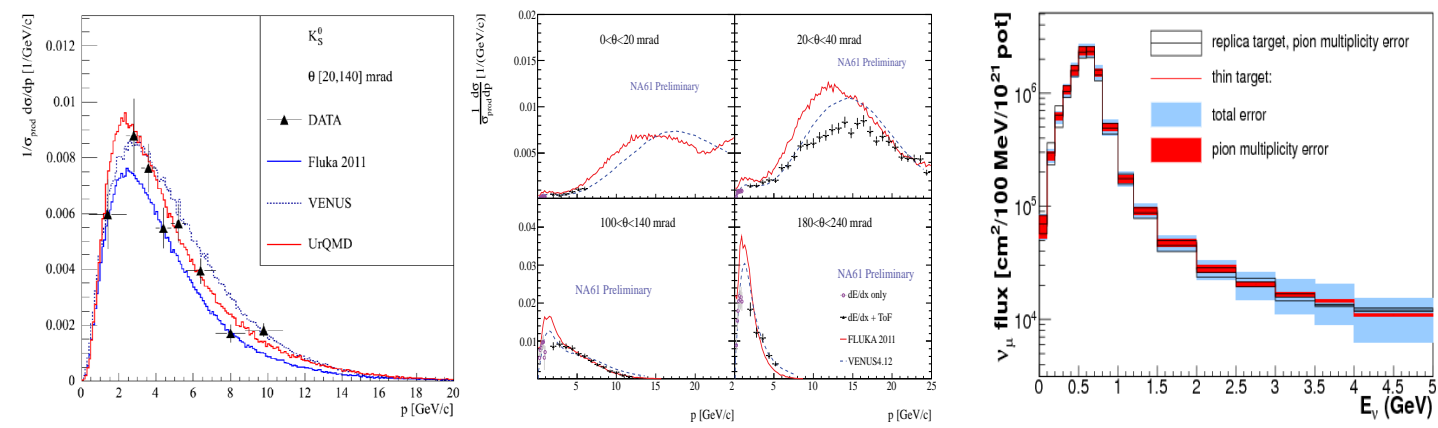

Figure 3: Left: production cross section of $K_{S}^{0}$ 's in a specific interval of the polar angle $\theta$. Center: production cross section of protons for several $\theta$ intervals. Data point markers indicate whether $d E / d x$ or $d E / d x+$ ToF information is used for the particle identification. Both $K_{S}^{0}$ 's and $p$ 's measurements refer to $p+\mathrm{C}$ interactions at $31 \mathrm{GeV} / c$. Predictions of different hadron production models are superimposed. Error bars indicate statistical and systematic uncertainties added in quadrature. The overall uncertainty $(2.3 \%)$ due to the normalization procedure is not shown Right: re-weighted $v_{\mu}$ flux at the far detector of T2K based on the NA61/SHINE thin target and replica target measurements.
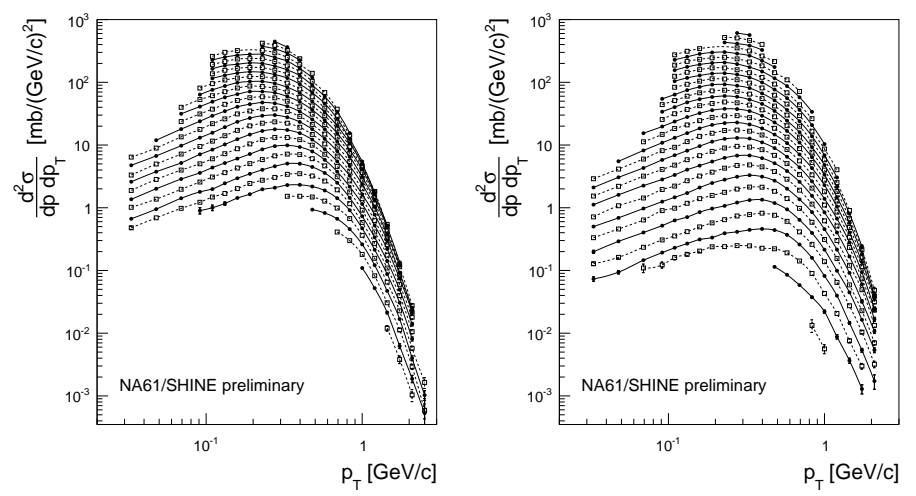

Figure 4: Inclusive $p_{T}$-spectra of charged hadrons produced in $\pi^{-}+\mathrm{C}$ interactions at $158 \mathrm{GeV} / \mathrm{c}$ for different values of the total momentum $p$. From top to bottom $p$ goes from 0.6 to $121 \mathrm{GeV} / c$ in steps of $\log p /(\mathrm{GeV} / c)=0.08$. Uncertainties include both statistics and systematic erros.

production cross section in $\pi^{-}+\mathrm{C}$ at both $158 \mathrm{GeV} / c$, shown in Fig. 4 , and $350 \mathrm{GeV} / c$. These data are already accurate enough to test the prediction of models used in the simulation programs.

Results on ion-ion physics The data taking for the NA61/SHINE ion program started with the energy scan of $p+p$ interactions. These data are needed to establish a small system-size base line for the results on collisions of light and medium size nuclei. The latter are particularly important in the search for the critical point of strongly interacting matter. First measurements of $\pi^{-}$spectra in $p+p$ collisions at 20,31, 40, 80, and $158 \mathrm{GeV} / c$ are available. In Fig. 5(right) the $\pi^{-}$transverse-mass $m_{T}$ spectra at mid-rapidity are compared with the spectra measured in central $\mathrm{Pb}+\mathrm{Pb}$ reactions by the NA49 experiment [6]. The observed differences could be due to transverse collective flow present in $\mathrm{Pb}+\mathrm{Pb}$ collisions and absent in $p+p$ interactions. The missing part of the $m_{T}$ spectrum is extrapolated using an exponential fit to the high $m_{T}$ tail. This allows to obtain the $m_{T}$ integrated rapidity spectra presented in Fig. 5(center). The spectra are well described by a sum of two symmetrically displaced Gaussian functions. When compared to $\mathrm{Pb}+\mathrm{Pb}$ data the widths of $\pi^{-}$rapidity distributions for $p+p$ are clearly narrower. The possible reasons are isospin effects and/or longitudinal collective flow in central $\mathrm{Pb}+\mathrm{Pb}$ collisions. The two-Gaussian fit allows 

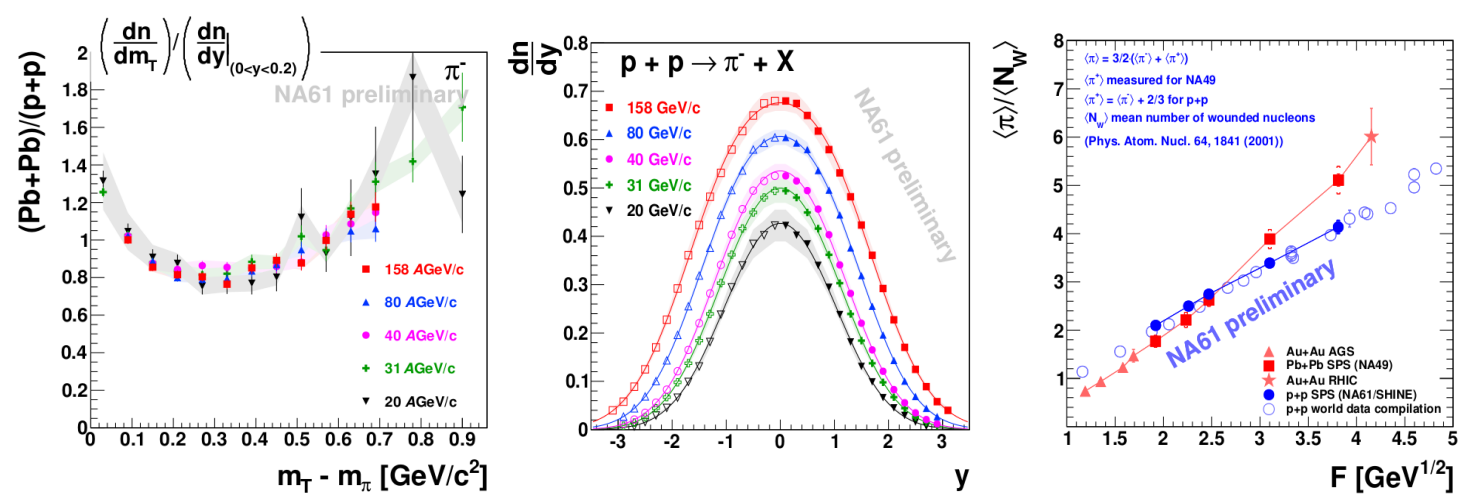

Figure 5: Right: $\pi^{-}$transverse-mass spectra for $\mathrm{Pb}+\mathrm{Pb}$ data divided by those for $p+p$ interactions. Shadow bands correspond to systematic uncertainty (mainly model based corrections). Center: $m_{T}$-integrated $\pi^{-}$ rapidity spectra fitted with a sum of two symmetrically displaced Gaussians. Right: Energy dependence of the mean pion multiplicity per wounded nucleon in full phase space $(4 \pi)$.
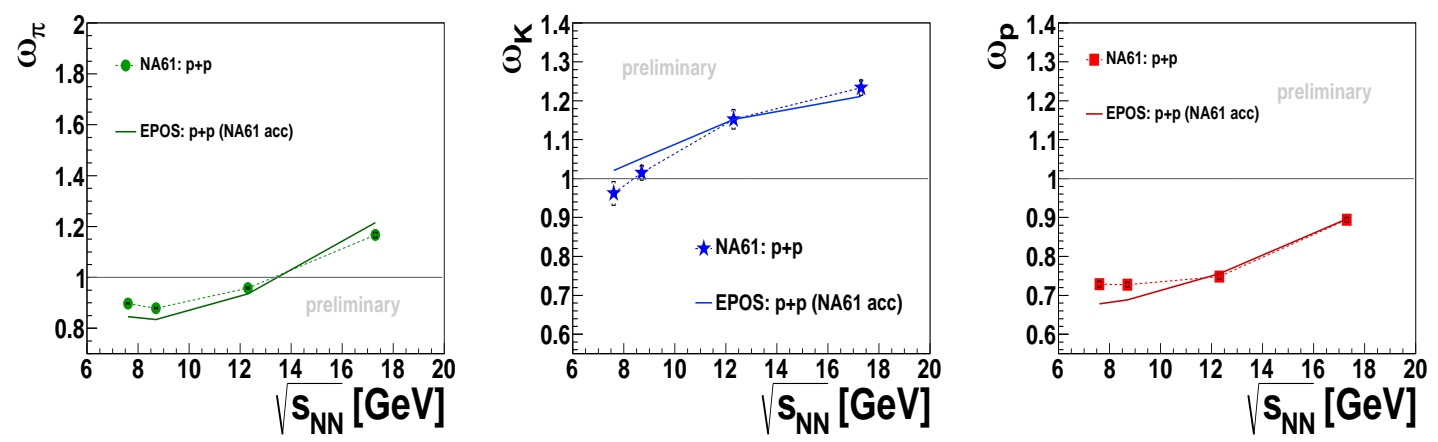

Figure 6: Preliminary results on the scaled variance of multiplicity distribution for $\pi, K$ and $p$ compared to predictions of the EPOS model. Statistical uncertainties are smaller than the symbol size. Systematic uncertainties are indicated by black brackets.

to extrapolate the rapidity spectrum and calculate the mean $\pi^{-}$multiplicity. Fig. 5(left) presents the kink plot, showing the total pion multiplicity normalized to the number of wounded nucleons versus the Fermi variable $F \approx s_{N N}^{1 / 4}$. Unlike $p+p$ interactions, in central $\mathrm{Pb}+\mathrm{Pb}$ and $\mathrm{Au}+\mathrm{Au}$ collisions a change of slope around $30 \mathrm{~A} \mathrm{GeV}$ is visible. Such an increase can be explained by an increase of the effective number of degrees of freedom when going from hadron gas to QGP as a consequence of the activation of partonic degrees of freedom. Preliminary results on $p_{T}$ and rapidity spectra of charged pions, negatively charged kaons, and protons were obtained in inelastic $p+p$ interactions at 40,80 and $158 \mathrm{GeV} / c$ hadrons for the study of the properties of the onset of deconfinement by looking for the kink, horn, step and dale structures [8] in collisions of light and intermediate mass nuclei $(\mathrm{Be}+\mathrm{Be}, \mathrm{Ar}+\mathrm{Ca}, \mathrm{Xe}+\mathrm{La})$. Preliminary results on multiplicity fluctuations of $\pi=\pi^{+}+\pi^{-}, K=K^{+}+K^{-}$, and $p=p+\bar{p}$ were obtained for $p+p$ interactions at $31,40,80$ and $158 \mathrm{GeV} / c$. The identity method [7] allows to obtain second and third moments (pure and mixed) of identified particle multiplicity distributions corrected for misidentification effects. From the first and second corrected moments of the multiplicity distributions, $N_{i}$ and $N_{i}^{2}(i=\pi, K, p)$, the 

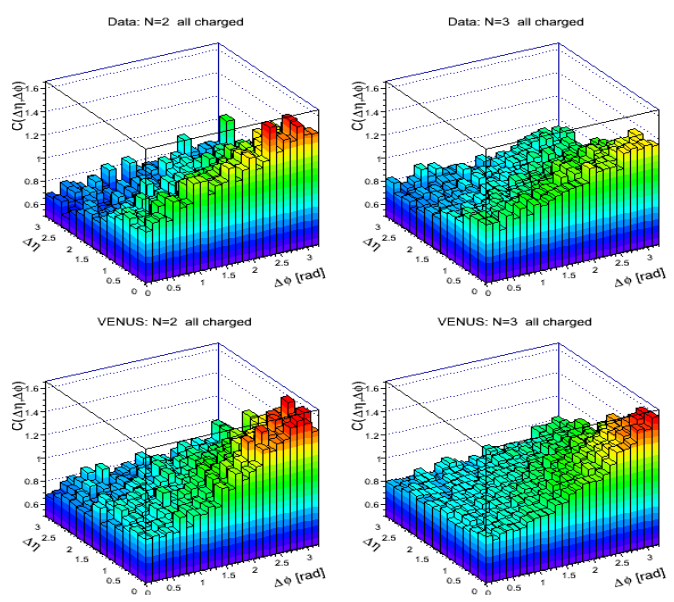

Figure 7: The two-particle correlation function for $p+p$ interactions at $158 \mathrm{GeV} / c$ for multiplicity of 2 and 3, for all charged particles. Upper row: results obtained from NA61/SHINE data, lower row: results obtained from the VENUS model.

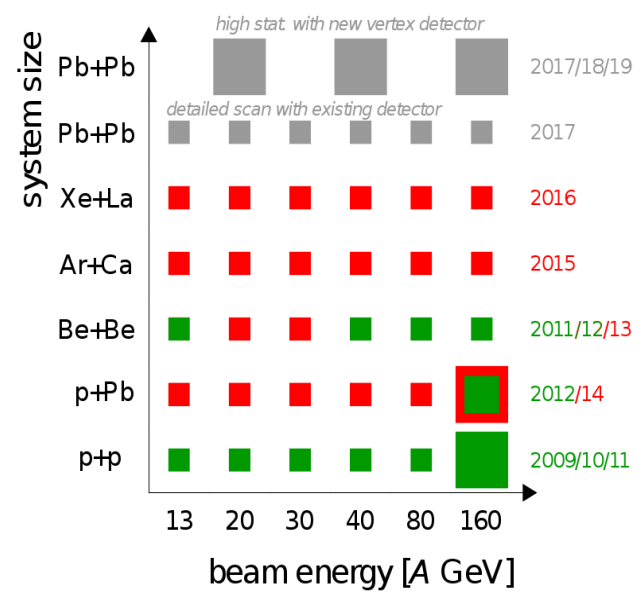

Figure 8: NA61/SHINE data taking plan for the ion program.

scaled variance is defined as $\left.\omega_{i}=\left(<N_{i}^{2}>-<N_{i}\right\rangle^{2}\right) /<N_{i}>$. This quantity equals 1 for Poisson multiplicity distribution and does not dependend on the number of wounded nucleons, however, it depends on wounded nucleon number fluctuations which may distort the comparison of $p+p$ data with results from nucleus-nucleus collisions. All studied scaled variances increase with increasing collision energy (Fig. 6). For kaons $\omega$ is above 1 for higher energies in agreement with EPOS predictions. This may be caused by the correlation of $K^{+}$and $K^{-}$production due to strangeness conservation. For protons $\omega$ is below 1, this is likely related to the fact that in most of events the number of final state protons is equal to the number of initial state protons (i.e. two). A comparison of NA61/SHINE results on multiplicity fluctuations (i.e. $\omega_{\pi}$ ) with the magnitude of fluctuations expected at the critical point [9] suggests that the systematic and statistical errors are small enough for a sensitive search of the critical point in NA61/SHINE. A two-particle correlation analysis was performed for $p+p$ interactions at $158 \mathrm{GeV} / c$. The correlation function $C$ is calculated as a function of the difference in pseudo-rapidity, $\Delta \eta$, and azimuthal angle, $\Delta \phi$, between two particles in the same event and is defined as

$$
\begin{gathered}
C(\Delta \eta, \Delta \phi)=\frac{N_{\text {mixed }}^{\text {pairs }}}{N_{\text {data }}^{\text {pairs }}} \frac{S(\Delta \eta, \Delta \phi)}{M(\Delta \eta, \Delta \phi)} \\
S(\Delta \eta, \Delta \phi)=\frac{\mathrm{d}^{2} N^{\text {signal }}}{\mathrm{d} \Delta \eta \mathrm{d} \Delta \phi} \quad M(\Delta \eta, \Delta \phi)=\frac{\mathrm{d}^{2} N^{\text {mixed }}}{\mathrm{d} \Delta \eta \mathrm{d} \Delta \phi}
\end{gathered}
$$

Two-particle correlations in $\Delta \eta, \Delta \phi$ were studied extensively both at RHIC and LHC. This method helps to disentangle different sources of correlations: jets, flow, resonance decays, quantum statistics effects, conservation laws, etc. Correlation functions were calculated with NA61/SHINE for multiplicities of 2, 3 (Fig. 7), 4, and 5, and separately for all charged, positively charged, and negatively charged particles. Results show interesting structures (Fig. 7). A significant maximum for all charged and positively charged particles at $(\Delta \eta, \Delta \phi)=(0, \pi)$ which comes probably from reso- 
nance decays and momentum conservation. For negatively charged particles this maximum is quite difficult to observe, because it is masked by a short-range correlations hill at $(0,0)$. Moreover an enhancement at $(\Delta \eta, \Delta \phi)=(0,0)$ coming from Coulomb and quantum effects is observable. Results from the VENUS model show similar structures except hills at $(0,0)$, because VENUS does not simulate Coulomb and quantum effects. In the near future there is a plan to repeat this analysis for the EPOS model, and then try to obtain $(\Delta \eta, \Delta \phi)$ correlations for pairs of identified particles produced in NA61/SHINE $p+p$ interactions.

Planned physics program and future prospects The NA61/SHINE data taking plan for the ion program is presented in Fig. 8. The energy scan with $\mathrm{Pb}+\mathrm{Pb}$ collisions is considered as a possible extension. This will allow to significantly decrease statistical and systematic uncertainties of mesurements on $\mathrm{Pb}+\mathrm{Pb}$ data and to reach significantly higher precision in establishing the system size dependence of hadron production properties, which is relevant for both the search for the critical point and the study of the onset of deconfinement. NA61/SHINE considers as well to extend the neutrino program with further data taking in order to perform hadron-production studies as required by the Fermilab and CERN (e.g. LBNO [10]) long baseline neutrino oscillation experiments. For the following years the plan is to perform measurements of hadron yields with proton beam momenta ranging from $9 \mathrm{GeV} / c$ to $120 \mathrm{GeV} / c$ on a number of target materials. The data would be relevant for MINERvA [11], NOvA [12] and the future Long Baseline Neutrino Experiment (LBNE) [13]. Dedicated pilot measurements in $p+\mathrm{C}$ interactions at $120 \mathrm{GeV} / c$ were taken during the on-going run as an initial step.

\section{References}

[1] K. Abe et al. [T2K Coll.], Phys. Rev. D 87 (2013) 012001 [hep-ex/1211.0469].

[2] N. Abgrall et al. [NA61/SHINE Coll.], Phys. Rev. C 84 (2011) 034604 [hep-ex/1102.0983]. N. Abgrall et al. [NA61/SHINE Coll.], Phys. Rev. C 85 (2012) 035210 [hep-ex/1112.0150].

[3] K. Abe et al. [T2K Coll.], Phys. Rev. Lett. 107 (2011) 041801 [hep-ex:1106.2822].

K. Abe et al. [T2K Coll.], Phys. Rev. D 85 (2012) 031103 [hep-ex:1201.1386].

[4] N. Abgrall et al. [NA61/SHINE Coll.], NIM A 701 (2013) 99-114 [hep-ex/1207.2114].

[5] J. Abraham et al. [Pierre Auger Coll.], NIM A 523 (2004) 50. T. Antoni et al. [KASCADE Coll.], NIM A 513 (2003) 490.

[6] S.V. Afanasiev et al. [NA49 Collaboration], Phys. Rev. C 66 (2002) 054902. C. Alt et al. [NA49 Collaboration], Phys. Rev. C 77 (2008) 024903.

[7] A. Rustamov, M.I. Gorenstein, Phys. Rev. C 86, 044906 (2012) [nucl-th/1204.6632].

[8] M. Gazdzicki and M. Gorenstein, Acta Phys. Polon. B 30 (1999) 2705.

M. Gazdzicki, M. Gorenstein, and P. Seyboth, Acta Phys. Polon. B 42 (2011) 307.

[9] K. Grebieszkow, Nucl.Phys. A 830 (2009) 547C-550C [nucl-ex/0907.4101].

[10] A. Stahl et al., CERN-SPSC-2012-021 (SPSC-EOI-007).

[11] D. Drakoulakos et al. [Minerva Collaboration], [hep-ex/0405002].

[12] D.S. Ayres et al. [NOvA Collaboration], [hep-ex/0503053].

[13] T. Akiri et al. [LBNE Collaboration], [hep-ex/1110.6249]. 